\title{
Research on the IFC Based Data Flow of OA System for Construction Projects
}

\author{
Zhao $\mathrm{Xu}^{1}$, Xiayan $\mathrm{Xu}^{1}$ and Nan $\mathrm{Chen}^{2}$ \\ ${ }^{1}$ Department of Civil Engineering, Southeast University, Nanjing, JiangSu \\ Province, China, 210096 \\ ${ }^{2}$ Department of Civil Engineering, SanJiang University, Nanjing, JiangSu \\ Province, China, 210000 \\ bernardos@163.com,xiayan_xu@163.com,15195906220@163.com
}

\begin{abstract}
Building information model (BIM) has become the mainstream in the contemporary architecture industry, which can integrate all construction project information during the life cycle, including all stages such as the survey, design, construction, delivery, use, operation, maintenance and so on. For BIM, IFC is a commonly used format which is an oriented file format with a data model to facilitate interoperability in the building industry. Besides, OA system is an efficient manager for information data while IFC standard is a kind of data expression standard for construction products. With data floworiented design of $O A$ system based on IFC, the management and application of IFC data with large number and complex structure are easily worked out, which makes sharing and utilization of resources during life cycle more efficient. This article deals with a detailed analysis of the IFC data format. According to the general principle of data flow-oriented design of OA system, the framework of data flow-oriented design based on IFC is proposed, as well as the framework of storage module and the extension method of the data dictionary based on IFC, which brings great convenience for model and big data management of enterprises.
\end{abstract}

Keywords: IFC, OA System, Workflow Design, BIM, Construction projects

\section{Introduction}

At present, leading a new direction of the development of building information technology, Building Information Modeling (BIM) has turned into technology application from an ideal concept, which brings construction industry new challenges and opportunities and requires a higher demand for decision-making and the application and management of information and data. On the platform of BIM software, drawing is replaced by BIM databases, which can contain all information throughout the life cycle of construction projects, including the stages of construction project planning, design, construction, operation, maintenance and demolition. As supported by mainstream BIM software, IFC standards is bound to be the basis of exchanging and sharing of BIM data, making it extremely important to do research on IFC standards for the development of construction industry. Meanwhile, the development of network technology has accelerated the replacement of traditional office with network office, making the reform of the construction industry very imperative. OA office systems applied in the construction industry, collaborative operation, efficient sharing and management of data and secure backups between different construction units can be achieved. Therefore, the combination of BIM technology and the OA office systems will be a qualitative leap in the field of building information. 


\section{Literature Review}

As an important research direction of information office and standardization, OA system and IFC standard has drawn much attention from researches. However, previous studies on OA and IFC always focus on the management methods of OA system itself and framework design of IFC standard. Johann (2009) proposed the concept of workflow and Workflow Management System [1].Shi et. al., (1999) suggested the concept of Workflow: among multiple participants, document, information or task which is delivered automatically in accordance with some predefined regulation, achieving the expected business goals [2]. Jiang et. al., (2000) discussed that the Office automation functions, including applications registering, reasonableness checking and opinions giving and exchanging, the workflow and organizational structure of $\mathrm{OA}$ should meet the requirement [3]. Lu and Gao (2012) analyzed the online business office automation system development process, office automation system requirement analysis, system function design and database design and considered that the database design should be on the basis of the system function data flow analysis and the logical structure of database system [4]. Lee et. al., (2011) proposed a set of metrics for quantifying the similarities and differences between IFC files [5]. Mohammad et. al., (2001) presented an object model for maintenance management of roofing systems as a case study to demonstrate the applicability of a proposed generic framework for integrating the maintenance management of built-assets. The model was built upon the Industry Foundation Classes (IFCs) (Releases 2.0 and 2.x) to define object requirements and relationships for the exchange and sharing of maintenance information between applications. They also designed several extensions to the IFC's including the representation of functional requirements, assessed conditions of objects, inspection and maintenance tasks, and libraries of non-project specific information [6]. Carlos and Lucio (2003) described an approach to automated integration of text documents in IFC compliant model-based systems [7]. Bogen and East (2011) described a repeatable process for efficient transforming design coordination view Industry Foundation Classes (IFC) models to multi-user visualization for Radiant, a popular 3D game engine [8]. Yeoh and Chua (2014) proposed a generalized, flexible and formal framework for representing various requirements to support the needs of the construction process using the Industry Foundation Classes (IFC) model specification [9]. Varieties of researches in the field bring great convenience to the integration of OA system and IFC standard in the building field. In order to offer new theories and methods for management and documentation of building information model and better implementation and application of building information model, this research aims to design a database structure of OA system based on IFC standard, offering convenience for model and big data management of enterprises

\section{Overview of OA Office System}

OA office systems which is also known as OA, is the abbreviations of Office Automation, referring to office automation or automated office. Based on the architecture model of "framework + application components+ platforms of function customization", the main part of OA management platform is made up of more than 30 subsystems, including information portals, collaboration, workflow and so on. OA automatic office systems is one kind of automatic office software with the B / S (Browser / Server) structure, which applies a three-tier structure of client browser -WEB application server database server and JAVA system structure with broaden coverage and maturity. Meanwhile, OA systems apply unified arrangement and centralized management to the administration with unified login, unified authority, and unified interface and unified basic data [10]. 


\section{IFC Standard and Data Format}

IFC standard is a kind of data expression standard for construction products, which is set by the International Alliance for Interoperability (IAI). IFC standard is essentially definition of building and engineering data, reflecting real-world objects. It adopts an object-oriented, standardized data description language, EXPRESS language, as data description language to define all used data. EXPRESS language makes description by a series of explanations, including Type, Entity, Rule, Function and Procedure [11]. The language definition and object description are mainly achieved by Entity in EXPRESS language and there are 653 entity types defined in IFC $2 \times 3$. Anentity description defines a data type of an object and its expression symbols, which is a description of the common characters of objects in the real world. Object characters are expressed by Type attributes and rules in entity definition. Entity attributes can be simple data types (numbers, strings, Boolean variables, etc.,) in EXPRESS, more than which are other entity objects. The same as other object-oriented languages, EXPRESS language can describe the inheritance and derived relationships between entities. Inheritance relationships between entities can be established by defining an entity as a Subtype or Supertype of another entity and Subtype can inherit properties of the Supertype. EXPRESS language supports multiple inheritances that one Subtype entity may possess several Supertypes at the same time. However, multiple inheritance is not supported in IFC standard so that all entity types only have one direct supertype. The building information model expressed by IFC standard data format can be divided into four functional levels: the Resource Layer, the Core Layer, the Interoperability Layer and Domain Layer [12]. Each level contains some information description modules and follows "gravity principle": each level can only refer to the information resources from the same or the lower information resources, but not the upper resources. So the lower resources won't be affected when upper resources change, which ensures the stability of information description.

It is of great significance to integrate IFC data format with OA system, which helps to achieve efficient management and sharing of data, collaborative operation between different people in different companies crossing geographies and safe storage and backup of building model data.

\subsection{The Spatial Structure of IFC File}

Standard format for each file will use a special method to decompose their file data into a number of sub-sets of data in order to find and organize files. IFC standard is also true that by building the spatial structure to manage the model data. The spatial structure of IFC file can be divided into five levels by the subordination of the project objectives, namely the Project, Site, Building, Storey, Building Element [13]. As shown in Figure 1.

According to IFC $2 \times 3$ implementation guidelines, the spatial structure of IFC standard data files can be divided into four classes. From the top to bottom are the domain layer, sharing layer, the core layer, resource layer [14], shown in Figure 2.

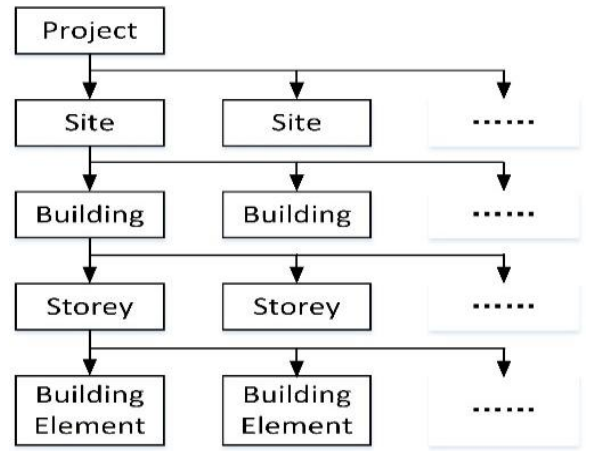

Figure 1. The Spatial Structure of the IFC File 


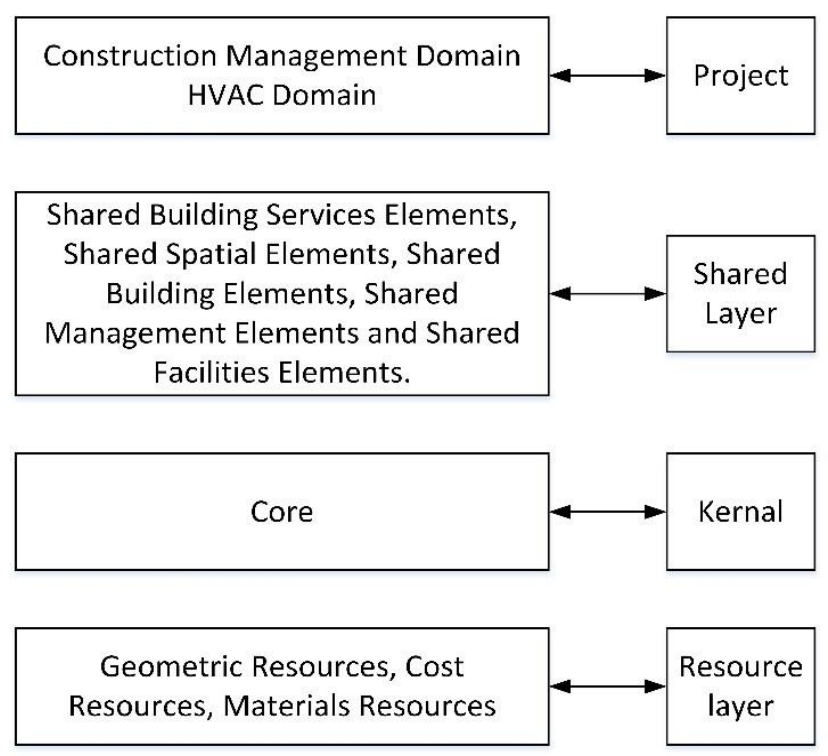

Figure 2. Four-Layered Structure of the IFC File

This article focuses on the data partition level of IFC data format based on the logical relationship.

1) Domain layer is the highest level in the spatial structure of IFC file format. The building information models which use or cite in the domain layer are independent of the core layer. The main role of the domain layer is to be able to go deep into the internal data in various application areas to form different specialized information, such as Construction Management Domain, HVAC Domain and other Domains and it can expand and develop constantly depending on the actual needs.

2) Shared layer is the secondary level in the spatial structure of IFC file format. The main function of this layer is serving the domain layer. Shared layer focuses on solving the problem of information exchange in the domain layer and makes the elements of each system more detailed, including the Shared Building Services Elements, Shared Spatial Elements, Shared Building Elements, Shared Management Elements and Shared Facilities Elements.

3) Core layer is the penultimate layer in the spatial structure of the IFC file format, which serves the shared layer and the domain layer. The main function of the core layer is to provide structure and concept of IFC files and organize the data belonging to the resource layer effectively into an organic one to reflect the structure of real model file.

4) Resource layer is the lowest level in the spatial structure of IFC file format and serves the other three layers. These data which are dispersed can be used in describing the corresponding basic information and applying to the entire IFC files. The resource layer Includes Geometric Resources, Cost Resources, Materials Resources, and so on.

\subsection{The Data Management Method of IFC Format File}

According to the standard requirements of IFC format, in order to use model data more interactively, the spatial structure of IFC files mentioned above in the core layer provides a logical relationship between a series of object types and further expansion of the IFC model. IFC data model is constituted by the entity, defined type, selected type, rules, functions, and property. IFC standard includes many entities, IFCROOT is the most important entity in the IFC root class. And the rest are derived from IFCROOT, which are divided into two categories: one is an independent entity for data exchange distributed 
in the framework layer, shared and domain layers with property named "GLOBALID". The other cannot be used to achieve data exchange independently, usually existing in the form of property derived from IFCROOT and not having a global identity characteristics, which are all distributed in the resource layer. Three subclasses were derived from the IFCROOT: IFCOBJECTDEFINITION, IFCRELATIONSHIP, IFCPROPERTYDEFINITION. These three abstract classes are important parts of IFC standard, derived from which enables IFC standard to describe specific building information model. If there is an "ABS" putting In front of the class name, it means that the class represents an abstract class.

Three subclasses: IFCOBJECT, IFCCONTEXT and IFCTYPEOBJECT are derived from IFCOBJECTDEFINITION. IFCOBJECT is the basic class for various architectural concepts. There are six subclasses derived from IFCOBJECT: IFCPRODUCT (such as walls, beams, columns), IFCACTOR (personnel), IFCGROUP (a bunch of objects), IFCPROCESS (process), IFCRESOURCE (resources) and IFCCONTROL (guidelines, norms).

IFCRELATIONSHIP is a special concept used to describe the relationship between physical objects andIFC builds the relationships between entities and concepts as abstract objects. Two objects must be linked by IFCRELATIONSHIP [5]. IFCRELATIONSHIP comprises six subclasses:

1) IFCRELASSOCIATES describes the relationship between objects and external resources.

2) IFCRELASSIGNS is used to describe the relationship when an object using the services provided by other objects.

3) IFCRELDECOMPOSES is used to describe the relationship between the objects constitution and decomposition.

4) IFCRELCONNECTS defines a connection between two or more objects in a certain way.

5) IFCRELDEFINES describes the object is defined by object type or object property.

6) IFCRELDECLARES is the inventory of object or property.

IFCPROPERTYDEFINITION is used to describe the class of object properties and two subclasses are derived from it: IFCPROPERTYSETDEFINITION and IFCTYPEOBJECT. The former is used to describe the properties of object except the shape. IFCTYPEOBJECT is used to describe the shape of the object.

The example for the spatial structure of IFC file:

\#25=IFCPROJECT('3sbLhr99fAYA_klA4ZJjov',\#5,'Tekla

Corporation',\$,\$,\$,\$,(\#11),\#24);

\#27=IFCSITE('01Ow6V1CfCvQz_jK7tETxJ',\#5,'Undefined',\$,\$,\#26,\$,\$,ELEMENT.,

$\$, \$, 0 . \$, \$)$;

\#3504=IFCRELAGGREGATES('Od_vU86HDDR9guJgQVPySB',\#5,\$,\$,\#25,(\#27));

\#29=IFCBUILDIBG('2Mm4beLdX6XOXRVK84WLJ\$',\#5,'Undefined',\$,\$,\#28,\$,\$,.E

LEMENT.,\$,\$,\$);

\#3505=IFCRELAGGREGATES('2NEdhkxhb4cQAJ9GQBzpgS',\#5,\$,\$,\#27,(\#29));\#3

504 describes the relationship between \#25 and\#27.\#3505 describes the relationship between\#27 and \#2. As shown in Figure 3. 


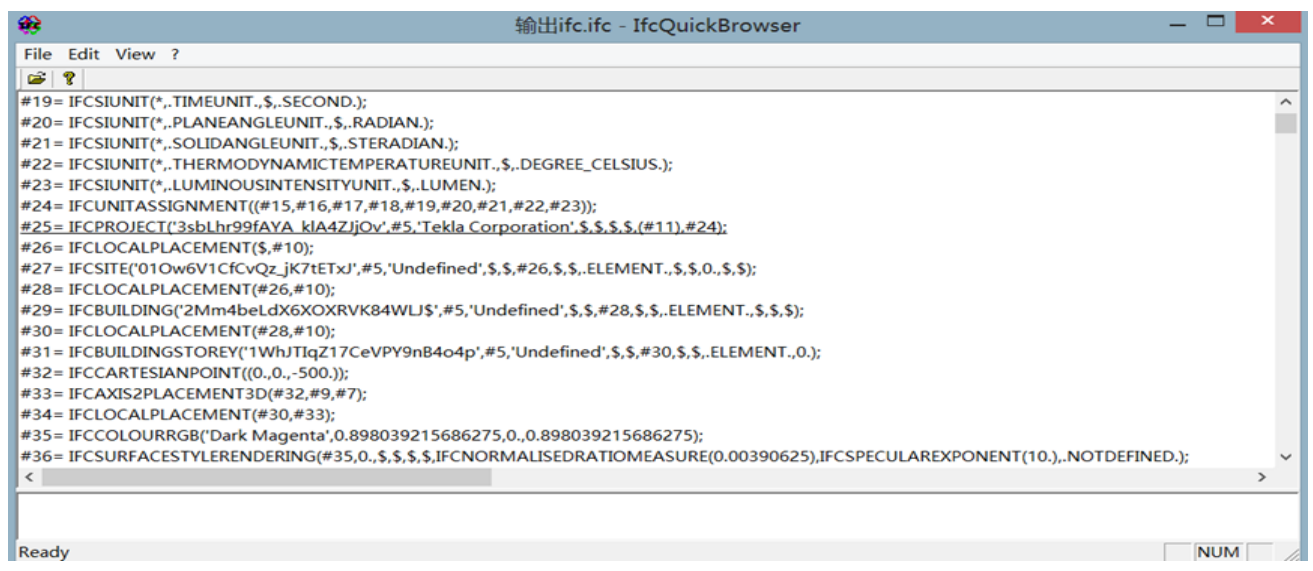

Figure 3. The IFC File in IFC Quick Browser

\#29=IFCBUILDIBG('2Mm4beLdX6XOXRVK84WLJ\$',\#5,'Undefined',\$,\$,\#28,\$,\$,.E LEMENT.,\$,\$,\$);

\#31=IFCBUILDINGSTOREY('1WhJTIqZ17CeVPY9nB4o4p',\#5, 'Undefined`\$,\$,\#30, \$,\$.ELEMENT.,0);

\#3506=IFCRELAGGREGATES( OfZULQ\$bj7FQ8WQvVsIyzT”,\#5,\$,\$,\#29,(\#31)); \#51=IFCCOLUMN( 1 JnC8b001EXZ4qC3KqC3Gq',\#5, 'COLUMN','HEA400','HEA 400`,\#34,\#50,`ID53c4c225-0000-4e86-3134-303534303434`);

\#857=IFCBEAM('1JnC8b001F234qC3Kq`,\#5, ‘BEAM`'HEA300`'HEA300`\#844,\#8 56,'ID53c4c225-0000-4f08-3134-303534303534');

\#3508=IFCRELCONTAINEDINSPATIALSTRUCTURE('2SYwqKqqXFZOqTKq8y uDh0”,\#5,\$,\$,(\#3223,\#3144,\#3079,\#3014,\#2944,\#2871,\#2800,\#2729\#2648,\#2551,\#2462, \#2359,\#2268,\#2179,\#2090,\#2001,\#1912,\#1823,\#1717,\#1648,\#1575,\#1502,\#1429,\#1351, \#1271,\#1188,\#1123,\#1058,\#993,\#928,\#857,\#782,\#717,\#652,\#587,\#522,\#457,\#392,\#327, \#262,\#197,\#127,\#51),\#31);

\#3506 uses IFCRELAGGREGATES to define the relationship between \#31 and \#29; \#3508 uses IFCRELCONTAINEDINSPATIALSTRUCTURE to define the relationship between \#51(a steel column), \#857(a steel beam) and \#31.

\section{Analysis of IFC-Oriented Data Flow of OA System}

\subsection{Overview of OA Data Flow Analysis}

With data flow out of automatic office system in the abstract, data flow analysis removes the specific organization, information carrier, processing tools and materials, examining the data processing mode of actual business from the aspect of data flow processes. Data flow analysis mainly consist of analysis on the flow, transform, analysis, processing and storage of information [15].

Existing data flow analysis is always achieved by multi-layered data flow diagram (referred to DFD). The specific approach is as follows: with data processing gotten in relevant research, draw a set of complete data flow diagram according to process order from flow chart. While drawings are sorted, the relevant data and reports, model, etc., should be checked [16].

The logical framework of data flow diagram is represented in Figure 4 


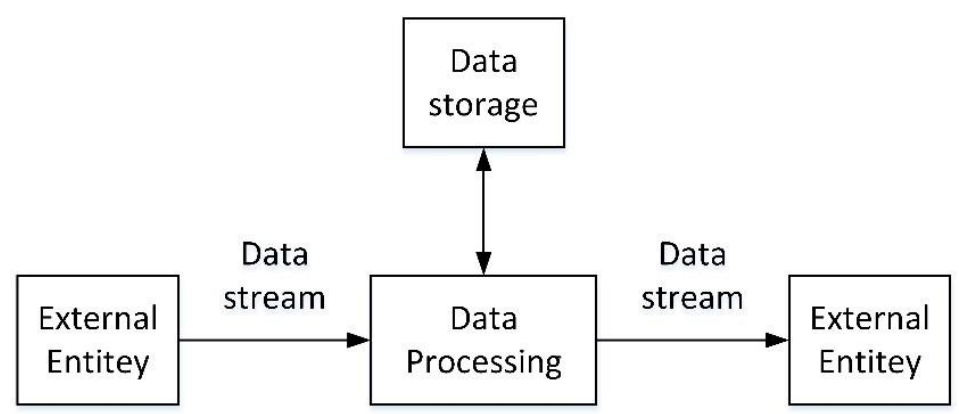

Figure 4. Logical Framework of Data Flow Chart

(1) The external entities in the figure refer to people and things outside but related to the system, which demonstrate the external source and destination of data and belong to the external part and interface of the system.

(2) Data flow refers to the input or output of processing function. The data flow is a tool to simulate the transfer process of system data. In the data flow diagram, data flow is represented by a vertical or horizontal arrow and arrows indicate the direction of data flow, with the name of data flow represented next to the arrows.

(3) The data processing refers to the processing of data logic which is also known as data conversion that is used to change the data value. And each processing includes data input, data processing, data output. In data flow diagram, data processing is represented by rectangle with rounded corners. The rectangle can be divided into three parts, in which the identification section is used to identify a feature, function description section is essential, and function executive section indicates that function is completed by whom [17].

(4) Data storage indicates the place of data storage, which is used to store data. System processing extracts data from data storage, and sends back the processed data to data storage [18].

\subsection{Overview of OA Data Flow Analysis}

Figure 5 shows the four-story frame structure of BIM: data layer, model layer, application layer and interface layer.

(1) The data layer is responsible for storage and management of information. Unlike traditional CAAD technology that uses pointless graphs such as point, line, plane and so on as information carriers, BIM technology uses object-oriented data organizing form and directly creates an instance of a wall that each instance has its attributes, including the position, size, composition and materials, etc., [19].Models like this carries more information than plans which consist of point, line, and plane, making it more suitable for computer to identify and process, as well as making sharing resources possible in different disciplines at different stages during life cycle.

(2) The model layer extracts the relevant information about specific professions as required and integrate various information from different professions into a model. The most common application is to extract information about buildings and structure for structural designers to make stress analysis. Besides, architectural, structural and device models can be integrated to detect conflicts of collision.

(3) The application layer is the expansion of BIM application. According to abundant information resources and readable organization of data, researchers can make data and 
statistics analysis to complete specific functions with powerful processing capabilities of computers.

(4) The interface layer provides an interface of Human-Computer Interaction, allowing participants and computers to identify model data, enabling all participants to read and manage building information during building life cycle.

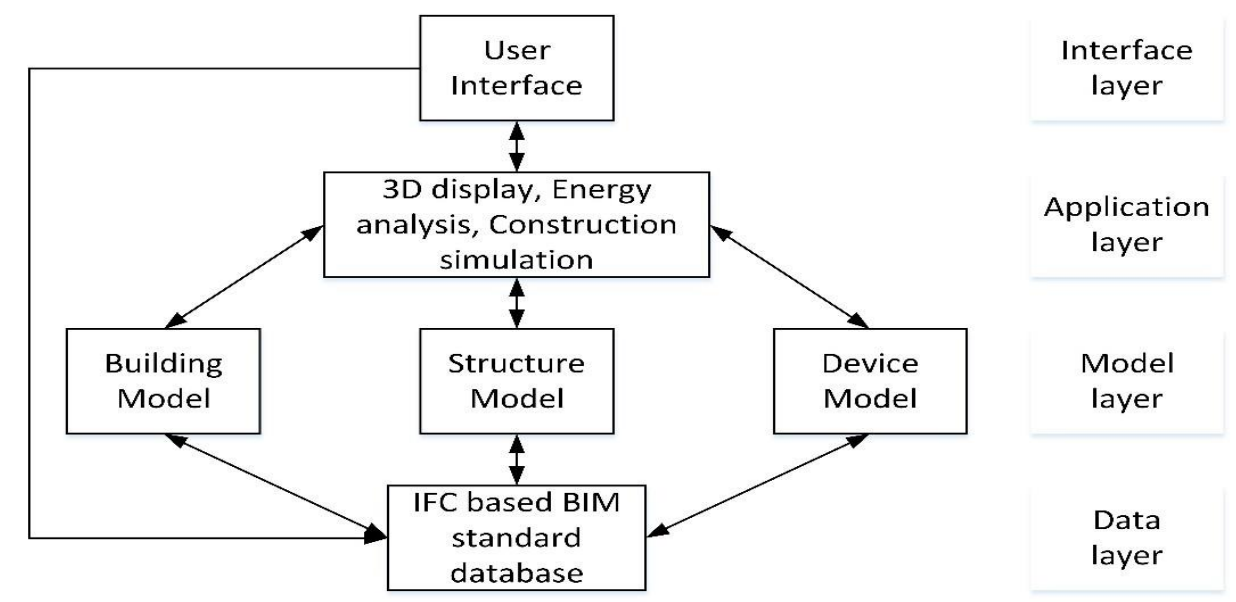

Figure 5. Framework of Building Information Model Based on IFC Standard

\subsection{Data Flow Design Combined with IFC Format}

5.3.1. Data Flow Design Combined with IFC Format: As shown in Figure 6, OA system structure combined with IFC data is divided into four levels.

(1) The user presentation layer: this layer is mainly to achieve interaction of system and people, specifically, who are ordinary OA client users in this system. For instance, designers in design organizations can enter into the landing page in the terminal equipment, and access the system to obtain the needed model information. Besides, they can also modify model files and upload to update the database. For the client administrator, he can get some other office information and modify the model permissions, as well as count the record of using and modifying models to get further systematic and efficient management.

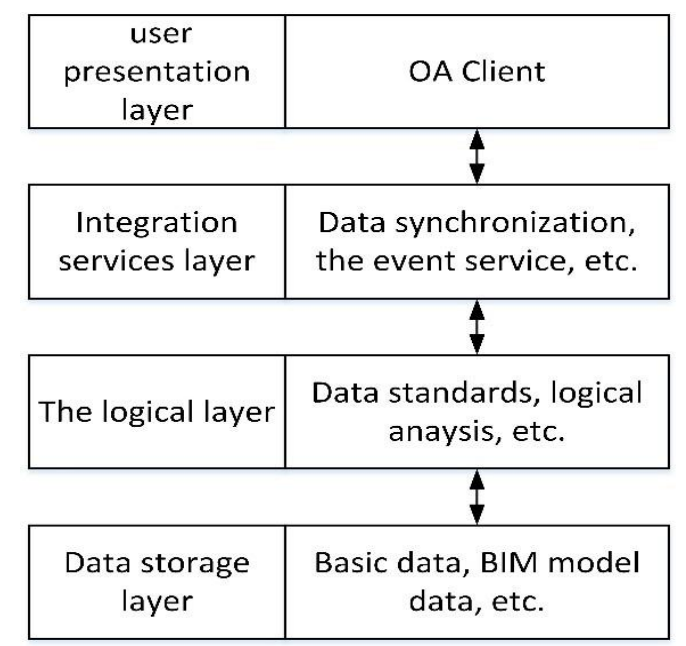

Figure 6. OA System Structure Combined with IFC Data 
(2) The integration services layer: integration services layer provides professional services functions, which is business-logic-layer-oriented. These functions include transmission services, event services, message queues services and data synchronization services, providing integrated system management services for this system.

(3) The business logic layer: this layer is to deal with processing requests sent from client in user presentation layer. It is beneficial for expansive requirements that encapsulate the processing of business in the business logic layer [20].

(4) The data storage layer: this layer is to store BIM model data, sharing information and published information data that will be used by systems, which uses database system with high performance and mass storage as supported software. The biggest difference between this system and the other OA systems is that the storage expansion of data from BIM model data in IFC format in data storage layer. That is to say, add IFC standard data module to data storage layer. The specific process is shown in Figure 7.

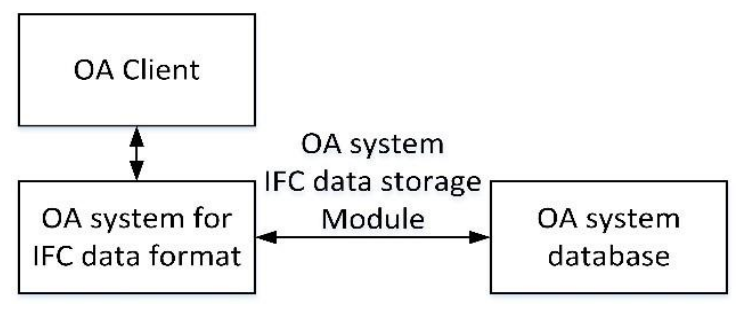

\section{Figure 7. Workflow of IFC Data Storage Module}

As shown in Figure 7, OA client user or administrator can input BIM in IFC data format to OA system. The system program will recognize this kind of data format file, and call the IFC data storage module in OA system. According to the IFC International standard data format, the IFC data is stored by data structure layers and integrated model information will be exacted. For example, extract construction model information from a building model, after which construction model is formed and stored in OA system database, in order to be user-friendly for construction engineers.

\subsubsection{Extension of Data Dictionary of OA System Based on the IFC Data}

Format: Recorded in a specific format, data dictionaries completely define and describe the content and features of each basic elements (data flow, processing, storage, and external entities) from data flow diagram of the system. It is one of important tools for structured systems analysis, as well as an important supplement and instructions of data flow. Data dictionaries are mainly used to describe the data flow, data storage, processing and external entities from data flow diagram [21]. Once established and sorted by numbers, the data dictionary is a dictionary available for people to consult, which will be used from systems analysis to system design and implementation. It is needed to keep the consistency and integrity of data in the establishment, amended and supplemented process of data dictionary. There are six types of entries in data dictionary: data, data structures, data flow, data storage, processing and external entities, of which different types need different attribute descriptions. For IFC-oriented OA system, the definition of IFC in IFC storage module in OA system is of great importance, to be specific, which means achieving the expansion of data dictionary based on IFC data format.

The Beam, Column, Door, Member, Plate, Slab, Wall, Window are divided into standard components and special components, respectively named Ifc Beam Standard Case and Ifc Beam. Standard component refers to the material or geometric constraints defining certain components. For instance, the member section of the path is a straight line or arc line. Building components also includes Chimney, Covering, Curtain Wall, 
Railing, Ramp, Ramp Flight, Stair, Roof, Shade Device and so on, which also defines component type In the IFC standard. Each building component has a corresponding Type entity, such as IfcBeamType. Common characteristics are used for members to share examples, such as the common definition of materials, cross-section, and property. Building components is a core part of the shared layer which contains both non-structural building components and structure components.

Building components are the same with other IFC entities, which describe their information with the property. Properties are divided into direct property, anti-property and export property. Direct property is an indicator of the amount or a collection of information. Anti-property is the definition of associated data and referential integrity and export property refers to the presentation of information by other properties. Direct properties: GlobalId, Name, Description, ObjectType, Tag. Export properties: OwnerHistory, ObjectPlacement (location information), Representation (geometric performance).Anti-properties: HasAssignments, HasAssignments, structural members cited (HasAssignments), component assembly (IsDecomposedBy, Decomposes), material definitions (HasAssociations), member classification (HasAssociations), type definitions (IsTypedBy), IsdefinedBy, component connection (ConnectedTo, ConnectedFrom), ContainedInStructure, space component reference (ReferencedInStructure), spatial boundary (ProvidesBoundaries), cover member (HasCoverings), whether contains openings (HasOpenings), projection information (HasProjections), whether filling openings (FillsVoids). The expression method for shape is the geometric properties belonging to export properties. An example of a shaped steel column to explain the method expressing the shape of the building components. Figure 8 and Figure 9 show the column in the Revit.

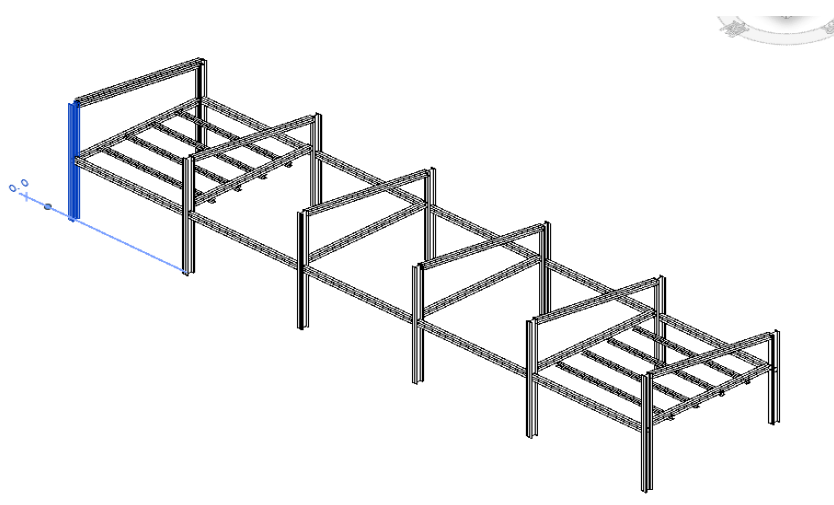

Figure 8. The Shaped Steel Column in Revit from a Whole View

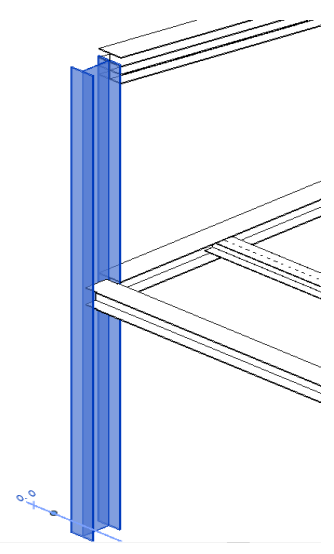

Figure 9. The Shaped Steel Column in Revit from a Local View 
\#51=IFCCOLUMN(`1JnC8b001EXZ4qC3KqC3Gq`,\#5, 'COLUMN','HEA400`'HEA 400`,\#34,\#50,`ID53c4c225-0000-4e86-3134-303534303434);

\#5=IFCOWNERHISTORY(\#3,\#4,\$,.NOCHANGE.,\$,\$,\$,1405931740);

\#34=IFCLOCALPLACEMENT(\#30,\#33);

\#50=IFCPRODUCTDEFINITIONSHAPE $(\$, \$,(\# 48))$;

\# 51 is the name of this root-shaped steel column, and other building components use the name when this column is cited. IFCCOLUMN is the keyword of this building components, which means that the building component is a column and the data in the parentheses is the real property. HEA400 is the name of descriptive type the column used. ID53c4c225-0000-4e86-3134-303534303434 is the unique identification for data integration and exchange. \# 5 is a reference to an instance of historical records. Records in this instance include when it's create, who create, what time it modifies, who modifies it and so on. \# 34 describes the position of this component. \# 50 is a reference to the shape of this component.

(1) The expression method for the Position of a column

In the IFC file format, there are two positioning methods of building components: one is called local positioning method, which is the relative positioning method in the world coordinate system and the other is in the whole (world) coordinate system coordinate description, known as absolute positioning methods. In the IFC format file, local positioning methods are mostly used for locating the building components [22]. The example of this column will be further explained in the expression method for the position of a column:

\# 34 = IFCLOCALPLACEMENT (\# 30, \# 33);

$\# 30$ = IFCLOCALPLACEMENT $(\# 28$, \# 10);

\# 33 = IFCAXIS2PLACEMENT (\# 32, \# 9, \# 7);

\# 34 is a reference to the other local coordinate. Local positioning method is therefore used a nested. Further expanded:

\# 6 = IFCCARTESIANPOINT $((0,0,0))$;

$\# 7$ = IFCDIRECTION $((1,0,0))$;

$\# 9=$ IFCDIRECTION $((0,0,1))$;

\# 10 = IFCAXIS2PLACEMENT3D (\# 6, \# 9, \# 7);

$\# 26$ = IFCLOCALPLACEMENT (\$, \# 10);

\# 28 = IFCLOCALPLACEMENT $(\# 26$, \# 10)

$\# 32$ = IFCCARTESIANPOINT $((0,0,-500))$;

\# 32 represents the Cartesian point coordinate values in the local coordinate system. The position is located by four nested, expanding in order. \# 9 describes this column at $\mathrm{Z}$ axis $(0,0,1)$ under the direction in the local coordinate system. \# 7 describes this column at Y-axis direction $(1,0,0$.$) direction in the local coordinate system. In the Cartesian$ coordinate system, a coordinate system is described, to indicate the general direction of the origin and three axes, but the direction of the vector $\mathrm{X} * \mathrm{Y}=\mathrm{Z}$ direction vector direction vector. In the IFC format, in order to reduce the amount of data, only two directions are shown. Since \# 34 is on the level of local positioning coordinate description, so according to the above method, it can be gradually expanded, which is a recursive definition of the method, until the local coordinate system is no longer a referenced to other local coordinate system. Then it is the absolute coordinate system. In actual, it need to convert constantly, until the member is drawn in the absolute coordinate system, then achieve the value of the origin, the $\mathrm{X}$-axis direction vectors, $\mathrm{Y}$-axis direction of the vector, the Z-axis direction of the vector. For column members, Y-axis direction under the local coordinate system is the real direction of the column members, but also the direction for the column to expand; Z-axis direction is the direction of cross-section height of the column. 
(2) The expression method for the shape of a column

Example for the expression method for the shape of a column is shown below:

\# 50 = IFCPRODUCTDEFINITIONSHAPE (\$, \$, (\# 48));

\# 48 = IFCSHAPEREPRESENTATION (\# 12, ‘Body’’'SweptSolid`, (\# 47));

\# 47 = IFCEXTRUDEDAREASOLID (\# 42 , \# 46, \# 9,7700);

\# 46 = IFCAXIS2PLACEMENT3D (\# 45, \# 44, \# 43);

$\# 45$ = IFCCARTESIANPOINT $((0,0,7700))$;

$\# 44$ = IFCDIRECTION $((0,0,-1))$;

$\# 43$ = IFCDIRECTION $((-1,0,0))$;

$\# 42$ = IFCISHAPEPROFILEDEF (.AREA, `HEA400` \# 41,300,390,11,19,27.);

\# 41 = IFCAXIS2PLACEMENT2D (\# 40, \# 39);

$\# 40=$ IFCCARTESIANPOINT $((0,0))$;

\# 39 = IFCDIRECTION $((1,0))$;

Defined by nesting, \# 48 shows the shape definition of this column. The Body describes the name and SweptSolid represents the type of the shape. \# 42 defines a crosssectional drawing and \# 45 defines the starting position of the drawing. Besides, \# 43and\# 44 define the direction of the extrusion and7700 defines the length of stretching. According to IFC standard, the definition of the cross section in the form of stretching includes: a closed circular cross-section, circular, L-shaped, T-shaped, U-shaped, Zshaped, etc., as well as any straight line without parameters [23].

\section{Conclusion}

With numerous participant units, long cycle of construction, many models made during construction process and other information, construction project is a very complex system. Therefore, how to manage a great deal of complicated information effectively has become popular research area in the field of construction. Building information model (BIM) has become the mainstream in the contemporary architecture industry, which can integrate all construction project information during the life cycle, including all stages such as the survey, design, construction, delivery, use, operation, maintenance and so on [15]. Besides, OA system is an efficient manager for information data. With data floworiented design of OA system based on IFC, the management and application of IFC data with large number and complex structure are easily worked out, which makes sharing and utilization of resources during life cycle more efficient.

This paper mainly studies the framework of BIM based on the IFC standard, as well as a further detailed analysis of the IFC data format. According to the general principle of data flow-oriented design of OA system, the framework of data flow-oriented design based on IFC is proposed, as well as the framework of storage module and the extension method of the data dictionary based on IFC. In order to offer new theories and methods for management and documentation of building information model and better implementation and application of building information model, this research could help to design a database structure of OA system based on IFC standard, offering convenience for model and big data management of enterprises.

\section{Acknowledgments}

The authors' special thanks go to all survey participants and reviewers of the paper, and appreciation to the National Science Council of P. R. C. for financially supporting this research (NSFC-71302138). 


\section{References}

[1] E. Johann, "Encyclopedia of Database Systems", Springer press, (2009), pp. 3545-3549.

[2] M. Shi, G. Yang and Y. Xiang, "Workflow Management System", Chinese Journal of Computers, vol. 3, (1999), pp. 325-334.

[3] J. Jiang. J, Chen, R. H. Yan and L. L. Xu, "A CSCW system for building reviewing by integrating GIS with OA”, Geo-spatial Information Science, vol. 3, no. 1, (2000), pp. 45-49.

[4] J. H. Lu and X. P. Gao, "Design and Realization of On-line Enterprise Office Automation System", Procedia Engineering, vol. 29, (2012), pp. 1809-1813.

[5] G. Lee, G. S. Won, H. Ham and Y. Shin, "Metrics for Quantifying the Similarities and Differences between IFC Files", Comput. Civ. Eng, vol. 25, (2011), pp.172-181.

[6] A. H. Mohammad, M. F. Thomas and J. Dana, "V.IFC-based Data Model for Integrated Maintenance Management", Comput. Civ. Eng, (2001).

[7] H. C. Carlos and S. Lucio, "Integration of Construction Documents in IFC Project Models", Construction Research Congress, (2003).

[8] A. C. Bogen and E. W. East, "Using IFC Models for User-Directed Visualization", Comput. Civ. Eng, (2011).

[9] K. W. Yeoh and D. K. H. Chua, "Representing Requirements of Construction from an IFC Model ", Comput. Civ. Eng, (2014).

[10] J. W. Tao, "The Analysis and Design of OA System", Yunnan University, (2013).

[11] L. Thomas, "IFC 2x Edition 2: Model Implementation Guide", (2005).

[12] K. N. Qiu and L. Wang, "The Implement Method of IFC Standard", Building Science, vol. 20, no. 3, (2004), pp.76-78.

[13] L. Thomas, “IFC 2x Edition 3: Model Implementation Guide”, (2009).

[14] K. N. Qiu and L.Wang, "IFC standard implementation method", Architecture Science, vol. 20 no. 3, (2004), pp.76-78.

[15] H. Y. Zhang and K. N. Qiu, "The examples for shape expression and the spatial structure of IFC", Civil Engineering Information Technology, vol. 2, no. 2, (2010), pp. 83-86.

[16] Y. F Dai and L. Dong, "The IFC standard for data expression and exchange", Architecture Science, vol. 24 , no. 1, (2008), pp. 9-14.

[17] H. Liu, "Design and Implementation of OA Office Platform Based on B/S Framework", Hebei University of Science and Technology, (2013).

[18] L. Y. Liu. "The Application and Research on Workflow in OA System", The Border Economy and Culture, no. 08, (2010), pp. 65-68.

[19] J. G. Huang, "Study on OA System Based on Database", Computer Knowledge and Technology, vol. 8, no. 15 , (2012), pp. 3497-3499.

[20] G. P. He, "BIM and BIM Software", Journal of Information Technology in Civil Engineering and Architecture, vol. 2, no. 4, (2010), pp. 110-117.

[21] X. B. Shi, "Design and Implementation of Office Automation System Based on B/S Mode", Ocean University of China, (2006).

[22] W. Ji, "The Development and Design of Office Automation systems", Computer Knowledge and Technology, vol. 8, no. 15, (2012), pp. 3746-3747.

[23] H. Y. Zhan and K. N. Qiu, "An Introduction to Shape Representation and Spatial Structure of IFC By Instances", Journal of Information Technology in Civil Engineering and Architecture, vol. 2, no. 2, (2010), pp. 83-86

[24] T. Su, W. Wang and Z. Lv, "Rapid Delaunay triangulation for randomly distributed point cloud data using adaptive Hilbert curve", Computers \& Graphics, vol. 54, (2016), pp. 65-74.

[25] W. Gu, Z. Lv and M. Hao, "Change detection method for remote sensing images based on an improved Markov random field", Multimedia Tools and Applications, (2015), pp. 1-16.

[26] Z. Lv, A. Tek and F. D. Silva, "Game on, science-how video game technology may help biologists tackle visualization challenges", PloS one, vol. 8, no. 3, (2013), pp. 57990.

[27] Z. Chen, W. Huang and Z. Lv, "Towards a face recognition method based on uncorrelated discriminant sparse preserving projection", Multimedia Tools and Applications, (2015), pp. 1-15.

[28] D. Jiang, X.Ying and Y. Han, "Collaborative multi-hop routing in cognitive wireless networks" Wireless Personal Communications, (2015), pp. 1-23.

[29] Z. Lv, A. Tek and F. D. Silva, "Game on, science-how video game technology may help biologists tackle visualization challenges", PloS one, vol. 8, no. 3, (2013), pp. 57990.

[30] D. Jiang, Z. Xu and Z. Lv, "A multicast delivery approach with minimum energy consumption for wireless multi-hop networks", Telecommunication Systems, (2015), pp. 1-12.

[31] V. Fu, V. Zhang and J. Jiang, "A Bayesian approach for sleep and wake classification based on dynamic time warping method", Multimedia Tools and Applications, (2015), pp. 1-20.

[32] Z. Lv, "Wearable smartphone: Wearable hybrid framework for hand and foot gesture interaction on smartphone", Computer Vision Workshops (ICCVW), 2013 IEEE International Conference on, IEEE, (2013), pp. 436-443. 
International Journal of $u-$ and e- Service, Science and Technology

Vol.9, No. 8 (2016)

[33] Y. Lin, J. Yang and Z. Lv, “A Self-Assessment Stereo Capture Model Applicable to the Internet of Things", Sensors, vol. 15, no. 8, (2015), pp. 20925-20944.

[34] J. Yang, S. He and Y. Lin, "Multimedia cloud transmission and storage system based on internet of things", Multimedia Tools and Applications, (2015), pp. 1-16. 